Revue d'histoire de l'Amérique française

ZWB REVUE D.HISTOIRE DE L'AMÉRIQUE FRANÇAISE

\title{
Henri Bourassa et la guerre sud-africaine (suite)
}

\section{James I. W. Corcoran}

Volume 19, numéro 1, juin 1965

URI : https://id.erudit.org/iderudit/302441ar

DOI : https://doi.org/10.7202/302441ar

Aller au sommaire du numéro

Éditeur(s)

Institut d'histoire de l'Amérique française

ISSN

0035-2357 (imprimé)

1492-1383 (numérique)

Découvrir la revue

Citer cet article

Corcoran, J. (1965). Henri Bourassa et la guerre sud-africaine (suite). Revue d'histoire de l'Amérique française, 19(1), 84-105.

https://doi.org/10.7202/302441ar d'utilisation que vous pouvez consulter en ligne.

https://apropos.erudit.org/fr/usagers/politique-dutilisation/ 


\section{HENRI BOURASSA ET LA GUERRE SUD-AFRICAINE * \\ (suite)}

Dans ce discours du 13 février 1900 - dont on peut lire un extrait (notre Revue, XVIII: 355) - Bourassa établit une distinction entre deux sortes de problèmes suscités par la participation du Canada à la guerre sud-africaine: la question de fait et la question de droit. Bourassa était convaincu, tout comme Goldwin Smith et bien des libéraux anglais dans tout l'Empire, que cette guerre constituait une action injuste et immorale, une pure et simple appropriation de territoire. Mais n'empêche que, légalement, cela ne concernait aucunement le Canada. Le danger de la participation militaire du Canada créait une autre sorte d'impérialisme, un effort pour réaliser une fédération impériale. Bourassa s'opposait à cette guerre qu'il considérait injuste et il croyait que la participation canadienne était un pas vers la fédération impériale.

Son nationalisme grandissant l'amena à insister fortement sur les arguments légaux et sur les conséquences futures que cette participation impliquerait dans les relations du Canada avec la Grande-Bretagne. Sans cacher, pour l'instant, qu'il considérait cette guerre injuste, il attendit la fin du conflit pour donner libre cours à ses sentiments :

Les Canadiens français, faisant en cela cause commune avec les libéraux anglais et avec l'humanité, ont condamné cette guerre odieuse et lâche, l'une des plus injustes que le monde ait connues depuis les pillages de Napoléon en Italie, en Espagne, en Hollande et dans les principautés allemandes. $^{19}$

* Voir notre Revue, XVIII : 343-356.

19 Henri Bourassa, Devant le tribunal de l'histoire (Montréal, 1903), 5 de la traduction de Goldwin Smith. 
Bourassa préféra discuter la question sud-africaine en rapport avec toutes ses conséquences pour le Canada et son rôle au sein de l'Empire. S'opposer à la participation canadienne était une position entièrement logique avec les principes du libéralisme. Bourassa prôna le maintien d'un empire décentralisé, il attaqua la fédération impériale comme une menace immédiate pour le Canada. Il discuta les aspects légaux et constitutionnels, moins explosifs que l'argument de la justice même de cette guerre. Politiquement, il aurait peut-être été plus rentable d'épouser la cause des Boers et de dénoncer la guerre, car plusieurs journaux du Québec sympathisaient avec les Boers.

Devant la résistance sublime d'un peuple préférant la mort à la soumission, le monde entier se demande pourquoi on veut exterminer cette nation héroïque, et si l'extermination de ce peuple, petit en nombre, mais grand par le courage et le caractère, n'est pas un crime plus odieux que le partage de la Pologne...20

D'autres attaques suivirent. Le danger de semblable opposition fut de faire soupçonner immédiatement et directement la "loyauté" du Canada français et de diviser racialement le pays.

Nous dénonçons celui qui (Laurier), pour la gloire de nous représenter au Parlement impérial, sacrifierait une position péniblement acquise et rendrait inutiles les luttes du passé. Avant de réussir, il devra soulever les pierres du sépulcre, arracher de leurs tombeaux nos morts illustres, jeter au souffle de l'ingratitude et aux vents de l'oubli la poussière des Viger, des Bédard, des Papineau, des Lafontaine, des Taché, des Cartier. Et après, il restera encore assez de vivants pour venger ces morts et pour punir celui qui aura trahi leur mémoire et compromis leur œuvre." 21

Plusieurs journaux du Québec demeurèrent pro-Boer durant toute la durée du conflit. "L'Événement, la Vérité, le Trifluvien, IX: 147 .

20 Robert Rumilly, Histoire de la Province de Québec (Montréal, 1930),

21 Rumilly, Histoire, IX : 45-46. 
le Monde Canadien, et le Pionnier ... exprimèrent ouvertement leur sympathie envers les Boers." 22 Un conservateur, Charles Thibault, prononça un violent discours politique à Sherbrooke:

Je blâme fortement Laurier d'appuyer l'Angleterre dans sa guerre injuste contre un petit peuple brave comme les Boers, qui luttent avec courage et patriotisme pour la liberté de leur pays. Je l'accuse de se faire l'instrument de Joe Chamberlain, un fou malcommode, et de Cecil Rhodes, un voleur. Cette guerre du Transvaal n'est qu'un acte de brigandage. Elle est faite au profit des compagnies qui veulent faire hausser la cote de leurs actions et augmenter leurs dividendes. ${ }^{23}$

De telles déclarations, la sympathie envers les Boers (dont la position était comparée à celle des Français au Canada) alliée au sentiment anti-impérialiste, conduisirent le Québec à l'anglophobie. Rt, dâns les joưrnaux de langue anglaise, on excitait la haine envers les Boers.

Cependant, en août et septembre, grâce à l'activité des agents britanniques qui trouvèrent un moyen d'expression dans l'agitation en faveur de la participation provoquée par le Montreal Star de Hugh Graham et les conservateurs dirigés par Sir Charles Tupper, l'opinion publique du Canada anglais ... fut soulevée contre les Boers. ${ }^{24}$

La barrière linguistique et les soupçons réciproques en plus de l'exaltation des sentiments créèrent rapidement une situation très dangereuse. Lord Minto, dont l'impérialisme ne fut jamais mis en doute, fit parvenir la dépêche suivante à Londres :

Les articles des principaux journaux de l'opposition en Ontario ont été odieusement malveillants, ne visant qu'à susciter de la haine à l'égard du Canada français. C'est parfaitement monstrueux ... Je crois moi-même que les Canadiens français sont très calomniés au sujet de leur déloyauté. Le Canada français ne veut pas être mêlé aux guerres impé-

22 Rumilly, Histoire, IX: 148.

23 Ibid.

24 Wade, 478. 
riales et il est tiède à cet égard mais dans la métropole, vous n'appelez pas déloyal un homme qui désapprouve la guerre. Ici, s'il n'est que tiède et Canadien français, il faut qu'il soit un rebelle. ${ }^{25}$

Mais traiter de la question de la justice de la cause des Boers en Afrique du Sud ne faisait qu'accroître la division raciale au pays. Bourassa lui-même devait bien le savoir et se rendre compte que le gouvernement Laurier ne pourrait indéfiniment résister; s'opposant à la participation canadienne, Bourassa contourna la question de justice et n'attaqua directement ni Laurier ni le gouvernement, mais, supputant les événements futurs, il croyait que la question de la fédération impériale était de loin la principale implication de la participation canadienne. L'enjeu, exprimé rationnellement, aurait certes, pu toucher les Canadiens d'origine française et d'origine anglaise. A une époque de surexcitation populaire, Bourassa ne prit pas le parti de l'aggraver davantage; il choisit de discuter les problèmes qui auraient des conséquences dans l'avenir, et de le faire sans appel ni aux sentiments ni aux passions.

La fièvre de la guerre, d'ailleurs, montait. Le 4 octobre 1899, le Globe publia une déclaration du Premier Ministre au sujet de la participation canadienne à n'importe quelle guerre de l'Empire.

Je comprends que selon la loi de la milice - et je puis dire que je l'ai étudiée avec assez d'attention ces derniers temps - nos volontaires doivent servir à la défense du Dominion. Ce sont des troupes canadiennes devant servir à la défense du Canada. (Le premier ministre expliqua alors que, dans une guerre où l'Angleterre combattrait une puissance dont la marine pourrait menacer le Canada, le gouvernement serait justifié d'envoyer des soldats outre-mer, sans attendre que nos côtes soient attaquées; et il ajoute:)

Le cas de la République d'Afrique du Sud n'est pas analogue. Le Canada n'est pas menacé, et même si nous voulons apporter une contribution militaire,

25 Wade, 486. 
je ne vois pas comment le faire. Et encore, comment le faire sans que le Parlement nous fournisse l'argent? Nous ne pourrions, en un mot, faire quoi que ce soit. En d'autres termes, il nous faudrait convoquer le Parlement... ${ }^{26}$

A peine la déclaration de Laurier était-elle publiée que lord Minto, le gouverneur général, recevait une dépêche de Chamberlain. La dépêche, portant la date du 3 octobre, avait déjà été largement diffusée dans la presse londonienne.

Le Secrétaire d'Etat à la Guerre et le commandant en chef désirent exprimer leur haute appréciation de la manifestation évidente de l'esprit patriotique du peuple du Canada, esprit patriotique démontré par l'offre de service en Afrique du Sud. Ils désirent fournir les renseignements suivants pour aider à organiser les forces offertes en unités compatibles aux exigences militaires. ${ }^{27}$

Le chef de l'opposition conservatrice, sir Charles Tupper, lui-même un impérialiste à tous crins, essayait de ramener son parti au pouvoir en faisant ouvertement appel aux sentiments impérialistes des Canadiens anglais.

Le chef de l'opposition a pris position (au sujet de l'article du Globe). Dans un télégramme qui ne s'est pas rendu à destination mais dont la teneur a été rendue publique, l'honorable gentilhomme a conseillé au gouvernement d'envoyer des troupes immédiatement, sans tenir compte du Parlement. Les deux positions étaient clairement établies et réflétaient bien les vieilles différences entre Torys et libéraux, en Angleterre et ici. ${ }^{28}$

Dans son discours aux Communes, le 13 mars 1900, Bourassa décrivit les événements. Dès l'ouverture des Chambres, d'ailleurs, il avait demandé que l'on produisît toute la correspondance échangée entre les gouvernements anglais et canadien.

26 Bourassa, Great Britain and Canada, appendix, XXXIV.

27 Bourassa, Great Britain and Canada, appendix, XXXV.

28 Bourassa, Discours du 13 mars 1900, Débats de la Chambre des Communes 1 (1797). 
Nous avons - ou du moins nous sommes censés avoir maintenant - toute correspondance échangée entre le Grande-Bretagne et les autorités coloniales; et que découvrons-nous? Nous découvrons qu'aucune offre n'a été faite à la Grande-Bretagne par le Canada, pas plus d'ailleurs que par Victoria, que par la Nouvelle-Galle du Sud, que par l'Australie du Sud, que par l'Australie de l'Ouest. En fait, deux gouvernements seulement... ont offert des troupes, soit la Nouvelle-Zélande et le Queensland, et nous verrons plus tard ce qu'il en est advenu. Au Canada le colonel Hughes a offert une assistance de caractère privé; transmise au gouvernement anglais, elle a été refusée poliment par M. Chamberlain. Alors, le même $M$. Chamberlain envoie une dépêche au gouvernement canadien, où il accepte une offre qui n'a jamais été faite, où il donne les règlements d'enrôlement, la date de l'embarquement, etc. Voilà une façon singulière de correspondre, quand la réponse arrive avant que la lettre ne soit écrite, mais tout ceci fait partie, je suppose, de la nouvelle politique et de la nouvelle diplomatie. ${ }^{29}$

On déclare la guerre le 12 octobre 1899 et le gouvernement canadien subit d'intenses pressions. Une offre d'assistance qui n'avait jamais été faite avait été publiquement acceptée. Et la guerre était en marche. D'autre part, le premier ministre, un Canadien français, avait déclaré, pas plus tard que la semaine précédente, qu'il était impossible d'envoyer des troupes, surtout sans la sanction du Parlement. Cependant, sans que les Chambres soient convoquées, des troupes devaient s'embarquer, pas plus tard que le 31 octobre 1899, selon les instructions jointes à la lettre de Chamberlain. La publication de cette lettre et de celle de sir Charles Tupper qui réclamait un envoi de troupes, à l'instant, sans recourir au Parlement, avaient surchauffé l'opinion publique. Le Globe décrivit exactement la position de Laurier.

Sa race et sa religion, l'exposant à la suspicion des provinces anglaises, le forcèrent à acheter son

29 Ibid., (1810). 
salut politique en capitulant sans condition devant le sentiment impérialiste. ${ }^{30}$

Laurier dut céder, mais il le fit de façon à préserver selon lui - l'indépendance future du Canada.

Dans ses Mémoires Bourassa décrit le caucaus libéral tenu chez Israël Tarte, le soir du 12 octobre.

Nous nous rendons chez M. Tarte où nous trouvons Napoléon Champagne, Belcourt qui était impérialiste. Laurier commence; il était merveilleux: "Notre ami Tarte nous a convoqués pour examiner la situation... Un certain nombre de gens veulent nous faire enrôler 10,000 hommes, organiser une armée pour aller en Afrique. D'autres comme notre ami Tarte ne veulent rien. Alors il s'agit de trouver une voie moyenne."

Je dis :- Avant de chercher une voie moyenne, tâchons de savoir qui a tort ou raison. Vous m'avez dit deux fois en trois semaines que vous n'enverriez pas de soldats en Afrique, et vous l'avez dit à Chamberlain, et il y a bien des raisons d'ordre constitutionnel; il y a aussi votre déclaration dans le Globe qui a paru il y a 7 jours. Comment allez-vous expliquer votre volte-face? ${ }^{31}$

Bourassa continua d'argumenter avec le premier ministre.

- M. Laurier, avez vous tenu compte de l'opinion de la Province de Québec? - Mon cher Henri, la province de Québec n'a pas d'opinions, elle n'a que des sentiments. ${ }^{32}$

Bourassa continua :

C'est parce que les circonstances sont difficiles que je vous demande de rester fidèle à votre parole. Gouverner, c'est avoir assez de cœur pour savoir, à un moment donné, risquer le pouvoir pour sauver un principe... Laurier soupira: - $\mathrm{Ah}$, mon cher ami, vous manquez d'esprit pratique. ${ }^{33}$

30 Rumilly, Histoire, IX: 153 (note).

31 Bourassa, troisième conférence, 62.

32 Rumilly, Histoire, IX: 121.

33 Ibid. 
Un ordre en conseil, daté du 13 octobre 1899, fut le résultat de la réunion.

Le premier ministre, vu le désir bien connu d'un grand nombre de Canadiens désireux de s'enrôler dans ces conditions, est d'avis que les dépenses modérées que comporteraient ainsi l'équipement et le transport de ces volontaires peuvent être immédiatement assumées par le gouvernement du Canada sans convocation du parlement, vu surtout qu'une semblable dépense, dans les circonstances, ne peut être considérée comme constituant un abandon des principes bien connus du gouvernement constitutionnel et de la coutume coloniale, ni interprétée comme établissant un précédent pour l'avenir. ${ }^{34}$

La lettre que Bourassa écrivit à Laurier, le 13 octobre 1899 , nous indique bien l'importance qu'il attachait aux questions à l'enjeu.

Cher M. Laurier,

Je vous ai dit hier soir que, lorsqu'on aura décidé de la politique d'intervention - qu'action ait été entreprise ou non -, j'accepterai que vous me dirigiez dans la voie à suivre: soit ma démission, soit discours et votes contre le ministère.

Après plus ample réflexion, je crois qu'il me faut reconsidérer cette décision et garder ma complète liberté d'action et le contrôle exclusif de mes opinions.

Je profite de l'occasion, ce matin, pour vous faire part de ma décision voulant garder envers vous la même franchise que j'ai employée depuis le début du problème. ${ }^{35}$

A ce stade-ci, une analyse des événements et de leurs implications est nécessaire. Nous avons une brillante illustration du premier ministre, homme politique à l'œuvre. Laurier, qui n'avait jamais douté de la justice de la guerre, croyait qu'en

34 Bourassa, Great Britain and Canada, appendix, XXXV.

35 Lettre de Henri Bourassa à sir Wilfrid Laurier, le 13 octobre 1899 (Papiers Bourassa). 
permettant à des volontaires, équipés et transportés aux frais du Canada, de se rendre en Afrique, il ne violait pas la loi de la Milice. Cette loi, telle qu'il l'interpréta le 4 octobre, défendait l'entretien outre-mer de troupes canadiennes. Le cas de ces contingents, formés de volontaires, était différent. Mais la riposte de Bourassa à cette prise de position, dans son discours du 13 mars 1900, vaut d'être signalée.

Maintenant, on fait grand état du fait qu'au lieu d'envoyer 2000 hommes déjà enrôlés, le gouvernement a enrôlé 2000 hommes et les a envoyés en Afrique du Sud. Je dois avouer que cette distinction est trop subtile pour mon esprit de rude paysan. ${ }^{36}$

Laurier approuva cette action inconstitutionnelle (qui engageait le gouvernement dans une politique impliquant une dépense des deniers publics sans l'assentiment du Parlement), en déclarant qu'une telle action ne devait pas être considérée comme un précédent. Cette clause du précédent (ou plutôt du non-précédent) semble s'appliquer aux frais et non à l'envoi de troupes canadiennes pour combat hors du pays. Bourassa, cependant, ne se montrait guère satisfait de cette distinction établie entre volontaires équipés par le gouvernement et soldats enrôlés; il croyait que cette prétention à l'absence de précédent ne visait qu'à camoufler deux accrocs au droit constitutionnel: soit la sanction du Parlement aux dépenses et à l'envoi de troupes canadiennes hors du pays. La section 79 de la loi de la Milice condamnait semblable dépense et le refus de participer aux guerres impériales faisait partie de la tradition canadienne.

Sir John A. Macdonald, le grand premier ministre conservateur, n'avait pas donné suite à pareille demande anglaise de fournir des troupes pour la guerre au Soudan, en 1885. Il avait déclaré, s'appuyant sur cette section 79 de la loi de la Milice, qu'il ne "sortirait pas Gladstone et Cie du trou dans lequel ils se sont eux-mêmes plongés par leur propre imbécilité.”37

36 Débats ..., discours de H. B., 13 mars 1900, 1, 1799.

37 Wade, 477. 
On avait donc un précédent et l'interprétation de ce précédent avait été confirmée par Laurier, le 4 octobre, dans sa déclaration au Globe. C'était précisément cette position que Bourassa cherchait à sauvegarder. Laurier abrogea implicitement cette position de MacDonald dans la formule de son ordre-en-conseil. Mais, quel que soit l'aspect légal du problème, le rôle traditionnel du Canada au sein de l'Empire en était modifié. Les conservateurs, canadiens aussi bien qu'anglais, furent prompts à voir dans l'envoi du contingent un précédent qui engageait l'avenir. L'objection présentée par Bourassa était simple: il s'opposait précisément à cet envoi pour ce qu'il modifiait les relations traditionnelles entre la Grande-Bretagne et le Canada sans le consentement du Parlement ou du peuple canadien.

Le 14 octobre, le gouvernement annonça la nouvelle: 1000 volontaires seraient équipés et transportés en Afrique du Sud, et ce, aux frais du gouvernement canadien. Arrivés à destination, ils seraient incorporés à l'armée britannique. On n'avait pas convoqué le Parlement.

Le 18 octobre 1899 Bourassa présenta à Laurier sa lettre de démission, qu'il avait rédigée aux bureaux d'Israël Tarte à La Patrie. Cette première condamnation publique de la politique de Laurier parut dans La Presse du 20. Quant à Tarte, sa critique de Laurier était maintenant aussi du domaine public, car, le 14 octobre, il avait pris position dans La Patrie dans des termes que Bourassa devait développer. Mais il demeurera Ministre des Travaux publics et ami intime de Laurier. Leurs relations étroites portent à croire que Tarte influença profondément la pensée de Bourassa. Cette allégation, et la tentative d'unir Laurier, Bourassa et Tarte dans un triumvirat de culpabilité et de déloyauté, était monnaie courante chez les conservateurs et Jans la presse impérialiste. "Tarte, toujours représenté par le Star comme l'inspirateur de Bourassa, fut voué aux gémonies dans la presse et dans les assemblées de Toronto." 37a Bourassa,

37a Rumilly, Histoire, IX: 143. 
agacé par ces rumeurs, en disposa dans un discours aux Communes, le 13 mars 1900 :

... mais le même journal annonça bientôt que le gouvernement avait décidé d'expédier un régiment. Je revins aussitôt à Ottawa, je rencontrai le premier ministre et le ministre des Travaux publics (M. Tarte) et, pour la troisième fois, je dis au premier ministre que je ne pouvais appuyer cette nouvelle politique de son gouvernement. C'était la veille du jour où le décret ministériel fut lancé. Et notez mes paroles, M. le président, c'était la première fois que je parlais au ministre des Travaux publics de cette question. Rien ne m'amuse comme entendre parler de la domination à laquelle on prétend que ce ministre m'a soumis. Je ne nie point les relations personnelles qui existent entre nous - et j'aurais tort également de nier que la plupart des opinions émises par le ministre des Travaux publics sur cette question se rapprochent beaucoup des miennes; pas toutes cependant. Mais, de fait, M. Tarte était absent à Paris, La Patrie, que beaucoup de gens désignent comme son organe, n'avait pas encore publié une seule ligne traitant de cette question, que j'avais discuté la situation avec le premier ministre, lui exprimant alors les opinions que j'émets aujourd'hui.

Ce qui fait précisément notre sympathie mutuelle est un trait particulier de notre tempérament; cette disposition nous est commune, mais elle me soustrait à tout contrôle, même à celui de l'honorable ministre: je veux dire un amour sans bornes de la liberté de pensée, de la liberté de parole et de la liberté d'action. ${ }^{38}$

La pensée que développe la lettre de démission de Bourassa n'a rien à voir avec l'attitude de Tarte; elle est bien plutôt l'exposé et le développement de la protestation du député de Labelle présentée au premier ministre, d'abord en août après la résolution de sympathie, puis encore au début d'octobre alors que la fièvre de la guerre embrasait le pays. 1815.

38 Débats ..., discours de Henri Bourassa, 13 mars 1900, 1: 1814- 
Papineauville, 18 octobre 1899.

Monsieur le ministre,

Dans une déclaration publiée le 4 octobre par le "Globe" de Toronto, vous avez dit, à propos de l'envoi de milices canadiennes au Transvaal: "Suivant mon interprétation de la Loi de la Milice, et je puis dire que j'ai étudié cette loi ces jours-ci, nos volontaires sont enrôlés pour la défense du Dominion. Ils forment des troupes canadiennes destinées à combattre pour la défense du Canada... Le Canada n'est pas menacé, et bien que nous puissions désirer envoyer des troupes, je ne vois pas comment nous pourrions le faire sans que le Parlement nous votât les deniers nécessaires? Nous ne pourrions rien faire. En d'autres termes, il nous faudrait convoquer le Parlement."

En face de cette déclaration, j'attendais la convocation immédiate des Chambres, où chacun des représentants du peuple aurait pu exprimer ses vues sur la situation très grave qui nous est faite en ce moment sans le consentement ni la participation de ceux dont vous tenez le pouvoir.

$\mathrm{Au}$ lieu du décret de convocation, les journaux de samedi nous apportent la nouvelle que le ministère fédéral a pris sur lui de constituer un corps de mille volontaires, de les vêtir, de les armer et de les expédier au Sud-Africain aux frais et dépens des contribuables de ce pays. Et cette démarche sans précédent s'accomplit, non à la demande du gouvernement impérial, mais en réponse à une dépêche du secrétaire des Colonies, acceptant des offres de services dont nous ne connaissons ni l'origine, ni les termes, ni le motif.

Si des offres ont été faites, d'où et de qui viennent-elles? L'Empire britannique est-il vraiment en péril, et réclame-t-il nos armes pour le sauver? Ou sommes-nous en présence d'une tentative de fédération militaire de l'Empire, projet cher à M. Chamberlain?

Voilà des questions que le peuple canadien a le droit de poser et d'entendre résoudre nettement avant 
de se laisser entraîner dans une guerre dont je ne veux apprécier maintenant ni les causes ni la légitimité. Il me suffit de dire aujourd'hui que cette guerre n'est pas le fait du peuple anglais, qu'elle a été condamnée d'avance par plusieurs hommes d'Etat les plus distingués du Royaume-Uni; qu'elle n'affecte en rien les intérêts propres au Canada ni les intérêts communs de la Grande-Bretagne et du Canada; que, de l'aveu des impérialistes les plus bruyants, elle ne met nullement en danger la sécurité de l'Empire.

L'arrêté ministériel qui décrète l'enrôlement et l'expédition de nos troupes réserve, paraît-il, l'avenir, et empêche cette action d'être considérée comme un précédent.

Le précédent, monsieur le ministre, c'est le fait accompli.

Le principe en jeu est l'axiome par excellence du libéralisme anglais, c'est la base même du régime parlementaire: NO TAXATION WITHOUT REPRESENTATION. Et l'impôt du sang constitue la forme la plus lourde des contributions publiques.

Il s'agit de savoir si le Canada est prêt à renoncer à ses prérogatives de colonie constitutionnelle, à sa liberté parlementaire, au pacte conclu avec la métropole après soixante-quinze ans de luttes, et à retourner à l'état primitif de colonie de la Couronne.

Il s'agit de décider si le peuple canadien sera appelé à prendre part à toutes les guerres de l'Empire sans que les portes du cabinet et du Parlement impérial lui soient ouvertes, sans même que ses représentants et son gouvernement soient consultés sur l'opportunité de ces luttes sanglantes.

Je ne consentirai jamais à appuyer cette politique rétrograde.

Citoyen britannique, fier de ses droits et jaloux de sa liberté, loyal à l'Angleterre et à son auguste souveraine, je suis prêt à payer de ma personne et de mes deniers, de ma parole et de mes actes, pour défendre le drapeau britannique dans toute l'étendue de la Confédération canadienne. 
Mais loyal avant tout, par-dessus tout et toujours au Canada, j'ai promis aux électeurs de mon comté de travailler au progrès de mon pays sans déroger à l'esprit fondamental de sa constitution. Pour accomplir cette promesse, j'ai donné mon appui à votre gouvernement, tant que vous êtes resté dans les limites que le peuple canadien vous a tracées. J'ai approuvé et j'approuve votre politique administrative. Mais je vois dans ce dernier acte l'inauguration d'une politique constitutionnelle que la majorité de vos partisans a toujours dénoncée, et sur laquelle vous n'avez jamais consulté ni le Parlement ni l'électorat.

Tels sont les principes que j'aurais posés, les opinions que j'aurais fait valoir à la Chambre des communes. ${ }^{39}$

Cette lettre présente les grandes lignes de l'argumentation de Bourassa. Il la développera et l'affirmera au cours de son opposition à la politique de Laurier, à l'intérieur et à l'extérieur du Parlement. Bourassa y discernait bien le véritable problème qui était une orientation, sans examen et sans discussion, vers la fédération militaire impériale. La lettre que Belcourt, un impérialiste canadien-français, écrivit à Bourassa, le 21 octobre 1899, fait voir l'une des apparentes faiblesses de l'argumentation du député de Labelle.

Le Canada, colonie autonome, libre de donner ou non, a librement choisi de faire à la mère-patrie un don de "sang et d'argent". [Et selon Belcourt, un don librement consenti, une action volontaire, n'implique pas un précédent entraînant plus tard une action obligatoire.] Vous proclamez qu'il faut condamner le gouvernement qui n'a pas réuni le Parlement avant d'agir. Techniquement vous pouvez avoir raison, mais ceci n'est-il pas un aspect bien mince et indigne de votre "better judgment". ${ }^{40}$

On ne peut que difficilement décrire le geste du gouvernement canadien comme un don volontaire: Laurier a dû

39 Rumilly, Histoire, IX: 126-128.

40 Lettre de M. Belcourt à M. Henri Bourassa le 21 octobre 1899 (Papiers Bourassa). 
changer dramatiquement sa politique, moins d'une semaine après sa déclaration au Globe, sur réception de la dépêche de Chamberlain; et il a dû le faire sous les pressions grandissantes de la presse impérialiste anglaise et devant l'impérialisme non déguisé du gouverneur général, de sir Charles Tupper et du parti conservateur. Malgré la clause référant à l'absence de précédent, la presse canadienne interpréta généralement le geste de Laurier comme le fit la Gazette de Montréal:

Une initiative a été prise; les recrues sont enrôlées: le navire de transport est nolisé... L'offre du gouvernement a créé un précédent pouvant servir de guide aux autres ministres placés dans les mêmes circonstances. ${ }^{41}$

Bourassa en était convaincu: ce que Belcourt appelait un don volontaire constituait un précédent qui entraînerait de continuelles contributions canadiennes au militarisme anglais, et ces contributions d'hommes et d'argent iraient augmentant. Ce qu'on disait un don volontaire menaçait de devenir une obligation continue, une taxe qui, bien que librement consentie, était exigée à un moment où le sentiment impérialiste, hystérique et déraisonnable, dominait l'opinion publique. Aucun homme, doué de sens commun, ne peut soutenir que le Canada a trouvé son compte dans la participation aux guerres de l'Empire. Même les deux réductions de tarifs (25\% en 1897 et $131 / 3 \%$ en 1900) en faveur de la Grande-Bretagne n'ont provoqué aucune faveur réciproque de la mère-patrie. Bourassa établit sans équivoque qu'il ne se sentait aucun attrait pour la fédération impériale.

Fidèle au principe fondamental du parlementarisme anglais, je combats d'avance toute tentative de participation aux guerres impériales sans droit de représentation. Ceci ne veut pas dire que j'accepterais un projet quelconque de fédération militaire de l'Empire, même avec droit de représentation. ${ }^{42}$

41 Rumilly, Histoire, IX: 129.

42 Rumilly, Histoire, IX: 133. 
Toutefois si le Canada contribuait à l'effort de guerre impériale, il avait droit - comme colonie autonome - d'être représenté aux Conseils de l'Empire. Dans l'opposition de Bourassa à la fédération impériale et dans son insistance sur la représentation du Canada aux conseils impériaux s'il contribue à la défense de l'Empire, nous retrouvons implicitement la conception qu'il se faisait de la dignité du Canada, nation autonome.

L'histoire a démontré les forces de décentralisation alors à l'œuvre dans l'Empire. Ces forces, Bourassa croyait qu'elles provenaient du libéralisme des "Little Englanders" et devaient en fin de compte faire avorter tout plan de fédération impériale. Il se rendait compte que l'Angleterre ne voudrait pas et ne pourrait pas accorder de participation aux colonies autonomes dans les questions touchant à l'Empire (ou seulement à l'Angleterre). Il espérait bien le faire entendre à ses adversaires: l'acceptation d'une fédération militaire impériale n'était rien de moins que le retour au statut de colonie de la couronne et l'obstacle à l'évolution naturelle du Canada vers la souveraineté politique. Bourassa réaffirmait donc la doctrine des "Little Englanders" et celle de la décentralisation à la face de l'impérialisme réactionnaire de Chamberlain.

Dans sa lettre de démission, Bourassa ne repousse pas la vague d'impérialisme avec un nationalisme aussi radical, mais il prône le maintien du statu quo, le maintien des libertés acquises, et le complet examen, par le Parlement, de tout geste qui pourrait modifier les relations entre le Canada et la mère-patrie. La pensée de Bourassa contient en germe l'idée d'un "Commonwealth" où les nations dépendantes de la couronne et régies par des institutions analogues poursuivraient ensemble leurs idéaux communs; non pas à l'intérieur de structures impériales rigides, mais selon les lois et institutions en vigueur, et pour le profit réciproque de chaque partie composante.

$\mathrm{Au}$ quatrième paragraphe de cette même lettre de démission, Bourassa demande réponse à certaines questions que le Canada n'avait pas suffisamment examinées, et qui l'avaient 
convaincu que la participation à cette guerre importait moins au Canada que le précédent établi dans les relations impériales. Un mince contingent de 1,000 hommes constituait une infime contribution à la force militaire anglaise en Afrique du Sud. Pourquoi, alors, Chamberlain a-t-il exigé ce contingent ? Pourquoi a-t-il pressé tellement le gouvernement d'apporter sa contribution ? Comme nous l'a révélé la lettre de Chamberlain à lord Minto, la question n'était pas de faire fournir des troupes par le Canada, mais de faire ratifier par le gouvernement une contribution militaire aux forces de l'Empire. Bourassa continua de développer ce thème dans sa campagne de réélection dans Labelle.

J'ai dit, et je répète, que j'ai donné ma démission, non parce que le gouvernement avait envoyé des soldats au Transvaal, mais parce que cet acte constitue un changement radical dans nos relations avec la Grande-Bretagne. ${ }^{43}$

"L'action du gouvernement est illégale et inconstitutionnelle; malgré les réserves de l'arrêté en conseil, un précédent est établi, et la fédération militaire acceptée; le Canada, qui n'a point participé aux guerres récentes de l'Angleterre en Afghanistan et en Egypte, ne doit et ne peut participer aux guerres qu'elle poursuivra encore aux quatre coins du monde. Les faits: rien n'est moins sûr que la justice de la cause anglaise dans cette guerre." ${ }^{44}$

Nous avons déjà mentionné que la participation canadienne au conflit sud-africain avait ranimé le conflit entre Français et Anglais au Canada. Nous avons le témoignage de l'impérialiste lord Minto: tout Canadien français que la guerre n'enthousiasmait pas était considéré comme traître dans les milieux anglais. Nous comprenons que l'impérialisme britannique, avec ses préjugés anti-français, pouvait difficilement soulever le Canada français. Bourassa aurait pu augmenter son prestige politique au Québec en flattant l'émotivité du Canada français

43 Rumilly, Henri Bourassa, 59.

44 Rumilly, Histoire, IX : 131. 
ou même en dénonçant l'injustice de la guerre et les souffrances du petit mais courageux peuple boer; mais il délaissa constamment ces arguments pour faire appel aux questions plus importantes, même si moins rentables émotivement, de l'évolution constitutionnelle et de la fédération impériale. Son anti-impérialisme pouvait avoir une résonance particulière chez les Canadiens français, mais sa position, fondée sur la conviction que l'Empire britannique est une institution basée sur le principe d'une dévolution de pouvoirs, est entièrement rationnelle. Sans l'atmosphère de jingoïsme et de préjugés raciaux qui prévalait, cette position eût pu être adoptée par un Canadien de langue anglaise. Question de fait, le plus fort appui de Bourassa, au Canada anglais, fut celui de Goldwin Smith de Toronto. Smith fut amené, par son libéralisme, à combattre la guerre sudafricaine comme injuste et la participation canadienne comme contraire à la raison. Il se peut que le libéralisme anglais à travers l'Empire ait enraciné Bourassa dans sa conviction que la guerre sud-africaine constituait un acte d'agression motivé par l'espoir de gains économiques. Mais une attaque contre la participation canadienne, venant d'un Canadien français et basée sur ces arguments, aurait été immédiatement rejetée par les Canadiens anglais. Ceci n'aurait servi qu'à isoler davantage le Canada français. Le 27 octobre 1899, Bourassa écrivit à Laurier :

J'ai réfléchi au problème pendant plusieurs jours, avant de vous faire parvenir ma démission. Je m'étais cependant préparé à cette éventualité depuis le 31 juillet, quand vous avez incité le parlement à adopter cette fameuse résolution. Je vous ai alors dit ce que j'en pensais. Je nie que ma démission ait été présentée sous l'impulsion du moment. C'était une action à laquelle j'avais réfléchi, l'action d'un homme qui a pesé les conséquences de cette crise... Je vais continuer d'appuyer la politique générale du gouvernement, mais je ferai tout ce qui sera en mon pouvoir pour enrayer cette vague d'impérialisme irrationnel que son action récente a créée et qu'elle menace de poursuivre. [Dans cette lettre Bourassa demande à Laurier si le parti libéral va présenter un candidat contre lui dans Labelle; 
ce serait alors, suggère-t-il, un désastre pour le parti. Il insiste cependant pour que le premier ministre se laisse guider par son devoir et non par la voix de l'amitié.] $]^{45}$

Bourassa avait écrit sa lettre de démission dans un esprit d'opposition aux desseins impérialistes lequel remontait à la résolution de sympathie. Dans sa réponse, Laurier attaqua sérieusement la position de Bourassa.

Permettez-moi de vous faire remarquer que le problème auquel vous vous confinez dans votre lettre ne sert qu'à éluder la véritable question. Si votre objection était que les subsides requis n'avaient pas été votés, rien n'aurait été plus facile que de régulariser une situation que vous approuviez en principe; mais, de fait, que serait-il arrivé si le parlement avait effectivement été en session? Vous auriez voté contre toute proposition visant à aider la GrandeBretagne dans sa guerre contre les Boers. Voilà, en vérité, la réelle question...

Dites-moi, quelle attitude voulez-vous que prennent les Canadiens français au sein de la Confédération? Qu'ils s'isolent, ou qu'ils marchent à la tête de la Confédération? Ils doivent choisir entre l'impérialisme anglais et l'impérialisme américain. Je n'ai aucunement l'intention de vous susciter de l'opposition dans Labelle et je déconseillerais toute atteinte de ce genre que mon parti voudrait faire.$^{46}$

Tout au long des hostilités, Laurier et le parti libéral ne combattirent à peu près pas Bourassa. Israël Tarte, organisateur du parti dans la province de Québec, décida de ne pas faire d'opposition à Bourassa, ni dans cette élection partielle ni aux élections générales de 1900. Le journal libéral du Québec, La Patrie, s'opposa tout aussi mollement à Bourassa. ${ }^{47}$

${ }^{45}$ Lettre de Henri Bourassa à sir Wilfrid Laurier, le 27 octobre 1899 (Papiers Bourassa).

${ }^{46}$ Lettre de sir Wilfrid Laurier à Henri Bourassa, le 2 novembre 1899 (Papiers Bourassa).

47 Renseignements obtenus de M. Laurier Lapierre, professeur au département d'histoire du Collège Loyola de Montréal. La thèse de doctorat de M. Lapierre porte sur Israël Tarte. 
Bourassa répondit à la lettre de Laurier.

Où et quand ai-je jamais parlé d'isoler la province de Québec et en quoi mon attitude s'oriente-telle dans cette direction?

J'ai dit et je répéterai que mon opposition se situe sur le terrain constitutionnel. Je ne m'objecte pas, comme vous semblez le croire, à ce que le gouvernement dépense quelques centaines de mille dollars pour envoyer ces soldats en Afrique. Ceci est le problème purement concret, dans son optique la plus étroite. Je m'oppose à cette dépense et à cet envoi de troupes parce que je vois dans cette action le premier pas sur la voie de l'impérialisme militaire - et le gouvernement ne possède pas le droit de nous engager dans cette voie sans consulter le parlement et le peuple. Ce bon Belcourt voulait me démontrer que je donnais une importance excessive à mon interprétation et qu'aucun précédent n'avait été établi. Sans mentionner les commentaires de la presse impérialiste, commentaires qui montrent que $\mathrm{j}$ 'avais raison, je crois que les discours de Hutton [commandant de la Milice] et de Lord Minto [Gouverneur général] peuvent nous éclairer suffisamment, aujourd'hui, sur l'interprétation .que les représentants officiels de l'Empire au Canada donnent à votre geste... vous avez participé au premier acte d'une révolution politique.

Quand avez-vous déclaré à votre parti, au Parlement ou ailleurs, que nous devions choisir entre l'impérialisme anglais et l'impérialisme américain ? Ce n'est sûrement pas dans vos discours auxquels j'ai applaudi et où vous affirmiez que notre constitution devait demeurer intacte.

Je n'ai jamais été et je ne suis pas en faveur de l'indépendance, du moins pour le moment, et pour longtemps encore. Il me semble que nous pouvons demeurer quelque temps dans l'état de transition qui existe maintenant. Chamberlain veut nous faire quitter cet état de transition. Ce mégalomane possède une idée fixe: passer à l'histoire comme le bâtisseur de l'empire. Il peut fort bien 
devenir le destructeur de l'empire, comme Morley le lui a si bien dit à Manchester.

Mais si, comme vous le dites, il faut choisir entre impérialisme anglais et impérialisme américain, je suis prêt à faire beaucoup pour préserver le lien britannique.

S'il faut altérer notre constitution, ceci doit être accompli par les parlements de Grande-Bretagne et du Canada, et d'après des propositions nettes, clairement définies, qui seront échangées entre les deux pays. D'ici là, gouverneurs et gouvernés, de Lord Minto et du général Hutton jusqu'au plus humble citoyen, doivent respecter même la lettre de la constitution.

Tout ce que je veux c'est séparer le problème constitutionnel de la crise d'hystérie qui a frappé notre pays, ou plutôt partie de notre pays. ${ }^{48}$

La critique de Laurier n'était pas dépourvue de tout fondement. Les faits indiquent que Bourassa trouvait surtout son appui auprès des Canadiens français. Son appel, cependant, s'adressait à tous les Canadiens, de quelque origine qu'ils fussent. La puissance du sentiment jingoïste et de la presse impérialiste - en grande partie dominée, au Canada, par des pairs de fraîche date - explique que les Canadiens anglais ne furent pas plus nombreux à supporter la position de Bourassa. Le rôle de l'opinion publique est un facteur essentiel à la compréhension de l'époque. Les deux citations suivantes, l'une de Goldwin Smith et l'autre d'un correspondant du Guardian de Manchester, décrivent la situation tout au long de la guerre.

“J'ai déjà observé qu'il n'y a ni discussion ni réelle connaissance des affaires sud-africaines au Canada," écrivit M. Harold Spencer au Guardian, alors qu'il décrivait la situation canadienne durant l'élection de 1900.49 Goldwin Smith fut encore plus péremptoire:

48 Lettre de Henri Bourassa à sir Wilfrid Laurier, le 4 novembre 1899 (Papiers Bourassa). 1901.

49 Debates (1901), I: 321. Discours de Henri Bourassa, le 12 mars 
Les clameurs ont enterré la liberté de parole au parlement. La presse, en presque totalité, a été le reflet servile de la presse jingoïste d'Angleterre. Ainsi, les principaux aspects du problème ne sont jamais parvenus à la connaissance du peuple canadien. ${ }^{50}$

Bourassa n'accepta pas cette assertion de Laurier, à savoir que le Canada n'avait que deux voies à suivre: celle de l'impérialisme anglais ou celle de l'impérialisme américain. A supposer que Laurier eût réellement entretenu cette conviction à l'époque, ses vues ont considérablement changé par la suite. Mais l'on doit se rappeler que, face à un tel choix, Bourassa aurait opté pour les liens britanniques. Sans ajouter créance, pour lors, à un tel dilemme, il insista sur le fait suivant: s'il faut opter, que ce soit de façon claire, après discussion ouverte de tous les aspects du problème, et non sous les pressions de la presse, des envoyés spéciaux de Chamberlain, de Rideau Hall et des bureaux de la milice. Cependant, Laurier ne voulait pas soumettre la question au parlement ni même y laisser débattre le sujet de la guerre. Les Chambres ne furent convoquées que le $1^{\text {er }}$ février 1900. Bourassa continua sa campagne dans Labelle, sans opposition libérale ni conservatrice. Il fut réélu le 7 janvier 1900 . Le 26, un second contingent de 1,000 Canadiens répondit à la demande de lord Minto, gouverneur général, et partit pour l'Afrique.

$$
\text { (à suivre) }
$$

JAMES I. W. CoRCORAN

(Traduction de MARC LA TERREUR)

55.

${ }^{50}$ Goldwin Smith, Devant le Tribunal de l'Histoire (Montréal, 1903), 